\title{
THERAPY
}

\section{Tissue engineering: harnessing stem cells in cartilage repair}

Cartilage engineering has the potential to treat degenerative joint diseases such as osteoarthritis (OA). Now, reporting in Science, Kristen Johnson and colleagues describe a small molecule, kartogenin, that selectively promotes the differentiation of mesenchymal stem cells (MSCs) into chondrocytes. The work provides new insights into chondrocyte biology and highlights a potential new therapeutic agent that might boost cartilage repair.

Johnson et al. screened 22,000 druglike molecules for their ability to induce chondrogenesis. Of these, one compound, kartogenin, promoted chondrocyte differentiation from MSCs, and protected articular chondrocytes from cytokineinduced damage in vitro. Furthermore, intra-articular administration of kartogenin at the early stages of the disease course promoted cartilage repair in both collagenase and surgery-induced mouse models of OA.

Next, the investigators sought to understand the mechanisms by which kartogenin exerts its effects. The researchers found that kartogenin bound to filamin A, in particular to the FC-1 fragment. A known binding partner of this fragment is $\mathrm{CBF} \beta$ (in its resting state), and kartogenin was shown to block this interaction. As a consequence, $\mathrm{CBF} \beta$ is activated and is thus free to translocate to the nucleus, where it can bind RUNX proteins to regulate transcription.

In support of this concept, nuclear localization of CBF $\beta$ was markedly increased in human MSCs treated with kartogenin. Furthermore,

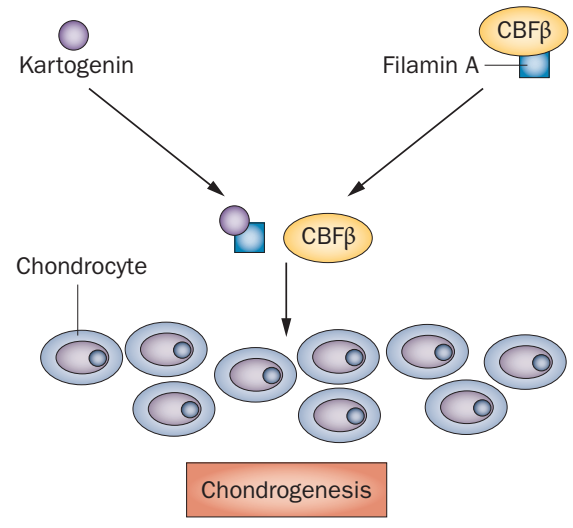

knockdown of CBF $\beta$ expression blocked kartogenin-induced differentiation of MSCs to chondrocytes, whereas CBF $\beta$ overexpression mimicked the effects of kartogenin on MSCs (that is, increased chondrocyte differentiation). Microarray analysis revealed that kartogenin affected gene expression in the RUNX1 transcriptional network, leading the authors to speculate that RUNX1 is the downstream effector of CBF $\beta$.

Further work is now planned to better understand the biology of kartogenin and the pathways it activates during cartilage repair. "Understanding the basic biology of stimulation of the endogenous healing events required for repair might allow us to move closer to developing an effective compound for clinical use," says Johnson.

\section{Katrina Ray}

Original article Johnson, K. et al. A stem cell-based approach to cartilage repair. Science doi:10.1126/ science. 1215157 